\title{
OCEAN TIDE LOADING AND RELATIVE GNSS IN THE BRITISH ISLES
}

\author{
P. J. Clarke and N. T. Penna \\ School of Civil Engineering and Geosciences, \\ Newcastle University
}

\begin{abstract}
Ocean tide loading (OTL) affects all parts of the British Isles to varying degree, causing peak-to-peak vertical displacements of up to $13 \mathrm{~cm}$ in South-West England over semi-diurnal and diurnal timescales. Lateral displacements are typically around one-third of the magnitude of vertical displacements at a point, so are also considerable for carrier phase GNSS surveying. Using a recent numerical ocean tide model, we predict that widespread residual displacements up to $\sim 1 \mathrm{~cm}$ remain in kinematic or shortoccupation static relative GNSS positions computed with respect to the nearest continuously operating reference station. Even if OTL is not modelled explicitly, these errors will be mitigated by network GNSS to an extent dependent on the number and location of reference stations used, and the adjustment or error interpolation scheme adopted in the processing. For a selection of error interpolation algorithms, we predict that throughout most inland regions of Great Britain and Ireland, network processing reduces the residual OTL error to within the expected kinematic GNSS system noise. However, pockets of OTL error exceeding $1 \mathrm{~cm}$ may remain, especially in coastal locations and in South-West Ireland, South Wales, South-West England, and the islands off the west coast of Scotland. Residual OTL error at semi-diurnal periods dominates that at diurnal periods. We derive a simple heuristic for estimating the magnitude of this error from a sample of observations at a locality, valid within a short window of the spring/neap tidal cycle, and show how the residual error may, if necessary, be mitigated by a suitable averaging scheme.
\end{abstract}

KEYWORDS: Global Navigation Satellite System (GNSS). Continuously Operating Reference Station (CORS). Network real-time kinematic (RTK) GNSS positioning. Periodic errors.

\section{INTRODUCTION}

Ocean tide loading (OTL) causes the periodic displacement of the Earth's surface as a result of the tidal redistribution of the oceans' weight that acts upon the seafloor. Even in the continental interiors, peak-to-peak vertical OTL displacements caused by the distant but widespread tides in the major oceans commonly exceed the centimetre level. Lateral displacements are not in direct proportion but are on average around one third of this magnitude. At island locations such as the British Isles that are surrounded by shallow continental shelf seas, the nearby shelf tides can exceed the few-decimetre open ocean tides by more than an order of magnitude; accordingly, local OTL displacements can exceed the decimetre level (peak-to-peak). With the existence of a complex coastline and bathymetry to perturb tidal currents, the phase lag of the local tide, and hence the magnitude of OTL displacement at a particular instant of time, can vary considerably over short distances.

OTL is therefore a source of potential error in geodetic positioning, in that it will displace a site from its conventional (long-term mean) position by a significant amount, over a period of several hours. The frequencies of OTL displacement follow those of the solid Earth and ocean tides: the vast majority of tidal power is dissipated at four semi-diurnal periods close to 12 hours and four diurnal periods close to 24 hours, with lesser but still potentially significant amounts at approximately fortnightly, monthly, and semi-annual periods (Table 1). OTL effects at these frequencies will 
combine constructively or destructively, according to the tidal cycles of which the fortnightly spring/neap cycle is dominant. Obviously, short-term averaging during the derivation of a single site position from anything up to a few hours of GNSS data will fail to remove OTL-related error; less intuitively, static positioning using longer session lengths will also leave residual OTL displacement error propagated to a number of long-period signals [1, 2, 3]. Absolute precise point positioning [e.g. 4] therefore requires that OTL displacements be modelled accurately epoch by epoch during coordinate estimation.

Table 1. Principal tidal constituents, their periods, and global amplitude of the forcing potential normalised to that of the M2 tide [after 5]. Tidal potential is latitude-dependent; hydrodynamic effects will further modify the ocean tide amplitudes and these figures only approximate their relative significance.

\begin{tabular}{ccc}
\hline Constituent & Period & $\begin{array}{c}\text { Normalised } \\
\text { potential }\end{array}$ \\
\hline $\mathrm{M} 2$ & $12 \mathrm{~h} 25 \mathrm{~m}$ & 1.00 \\
$\mathrm{~S} 2$ & $12 \mathrm{~h} 00 \mathrm{~m}$ & 0.47 \\
$\mathrm{~N} 2$ & $12 \mathrm{~h} 39 \mathrm{~m}$ & 0.19 \\
$\mathrm{~K} 2$ & $11 \mathrm{~h} 58 \mathrm{~m}$ & 0.13 \\
$\mathrm{~K} 1$ & $23 \mathrm{~h} 56 \mathrm{~m}$ & 0.58 \\
$\mathrm{O} 1$ & $25 \mathrm{~h} 50 \mathrm{~m}$ & 0.41 \\
$\mathrm{P} 1$ & $24 \mathrm{~h} 04 \mathrm{~m}$ & 0.19 \\
$\mathrm{Q} 1$ & $26 \mathrm{~h} 52 \mathrm{~m}$ & 0.08 \\
$\mathrm{M}_{\mathrm{f}}$ & $13.66 \mathrm{~d}$ & 0.11 \\
$\mathrm{M}_{\mathrm{m}}$ & $27.32 \mathrm{~d}$ & 0.06 \\
$\mathrm{~S}_{\mathrm{sa}}$ & $182.62 \mathrm{~d}$ & 0.05 \\
\hline
\end{tabular}

OTL displacements can be modelled by convolving a numerical ocean tide model, which describes the spatially varying surface mass load at a number of tidal frequencies, with a solid Earth model describing the response to a unit load. Typically, a radially symmetric, non-rotating, elastic, isotropic Earth model is used [6]. A number of computer programs are available to carry this out, for example SPOTL [7], GOTIC2 [8], CARGA [9] and OLFG/OLMPP [10]; the latter is used in the popular web-based OTL computation service http://www.oso.chalmers.se/ loading/. It has long been considered that uncertainties in the numerical ocean tide models dominate the error budget of OTL displacement computation [e.g. 9], especially near shallow seas where modelling inaccuracies are greatest, although near to all coastlines the effects of tidal model grid cell size and coastline definition are also important [11]. As an illustration of the former uncertainty, Figure 1 compares the absolute magnitude of vertical OTL displacements in the British Isles predicted from one modern numerical ocean tide model, along with the root mean square magnitude of the difference between five such models and their mean, for the largest semi-diurnal (M2) and diurnal (K1) constituents.

The majority of precise GNSS positioning performed in mapping, engineering and scientific surveys is carried out in relative mode, typically forming the doubledifference carrier phase observation between pairs of satellites and receivers so as to 

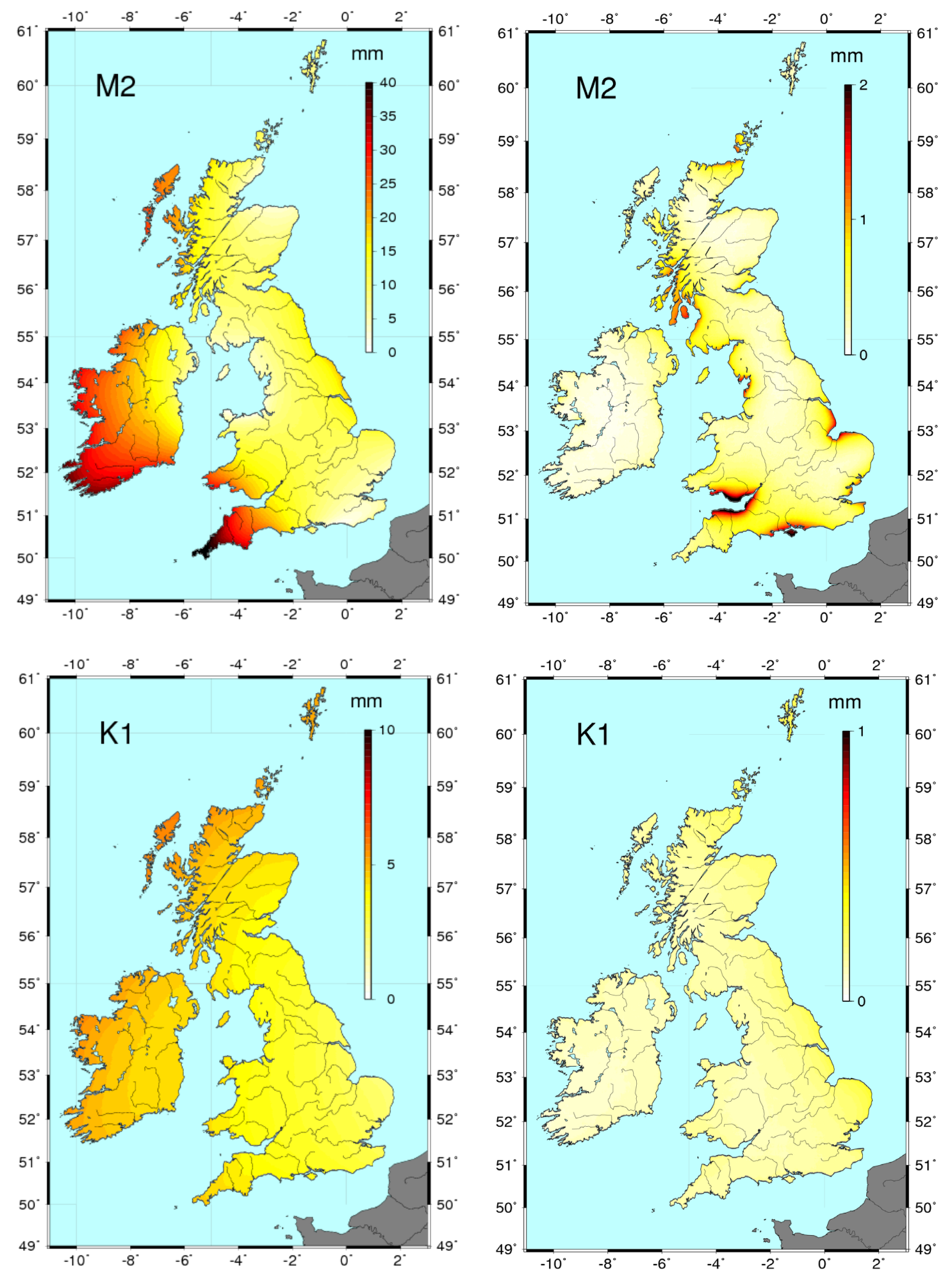

Fig. 1. Amplitude of vertical OTL displacements predicted from the FES2004 [12] numerical ocean tide model (left) and root mean square of the magnitudes of the complex-valued differences between the amplitudes and phases of this model, the CSR4.0 [13], GOT00.2 [14], NAO.99b [15] and TPXO7.1 [16] models, and the mean of all models (right). Top: M2 constituent; bottom: K1 constituent. Note that the scales vary. 
mitigate satellite orbit, atmospheric, antenna, and clock errors. It is often assumed, particularly in commercial software which often has no OTL displacement modelling capability, that this differencing will virtually eliminate OTL-related errors. We will show later that this is not necessarily the case, but because the OTL effects at the two ends of the baseline differ, it will, to first order, reduce the residual error to an amount very closely approximating this difference. In this paper, we demonstrate the level of residual OTL-related error that could be experienced by a user in the British Isles using national or commercial geodetic infrastructure to adopt relative GNSS, whether kinematic or short-occupation static, and whether real-time or post-processed. In this context, "short" refers to sessions of up to around an hour, i.e. much less than tidal periods, over which a batch least squares solution will recover the true average position within the window [17], at least when ambiguities are resolved, without any errors due to propagation effects as predicted [1] and observed [2] for longer sessions.

At present, there are around 130 continuously operating reference stations (CORSs) for GNSS in the British Isles (Figure 2), with the vast majority of these operated by the national survey agencies Ordnance Survey, Ordnance Survey of Northern Ireland (OSNI, now part of Land and Property Services), and Ordnance Survey Ireland (OSI). GPS, and increasingly GNSS, data from these survey agency sites are available to the public with latencies of around an hour. In addition to these major providers, there are some stations in mainland Britain operated by universities and government scientific agencies, which together with the national survey agency sites contribute to the British Isles continuous GNSS Facility BIGF (http://www.bigf.ac.uk/) with latencies ranging from hours to weeks; and some commercially-operated sites available to subscribers in near real-time. These CORS networks can be used to compute relative GNSS coordinates linked to a national or international reference frame.

Real-time kinematic (RTK) GNSS is increasingly used, especially in commercial surveys, because it reduces total operator time. Furthermore, in the British Isles (as in many other locations) there exist commercial providers of RTK reference station data delivered via a mobile telecommunications link, which removes the need for a surveyor to operate his or her own reference station with its attendant costs. An additional advantage is that these RTK services are provided in network mode $[\mathbf{1 8}, \mathbf{1 9}$, 20], which allows relative accuracy to be maintained at greater distances from the nearest reference station because error models can be interpolated within the network. Currently there are two service providers, Leica Geosystems "SmartNet" and Trimble "VRS". In mainland Britain, Network RTK services are provided overwhelmingly using data under licence from the Ordnance Survey active GNSS network, although SmartNet operates a limited number of additional reference stations. In contrast, in Ireland the SmartNet solution uses predominantly OSI and OSNI reference station data with some additional sites, whereas the VRS solution uses a wholly independent network with similar station spacing to the other three Irish networks combined.

\section{PREDICTION OF RESIDUAL OCEAN TIDE LOADING ERROR}

The residual ocean tide loading error in a relative GNSS solution, whether instantaneous or averaged over a period very much shorter than the tidal timescale, will depend on the mapping of OTL displacements into the differenced carrier phase observations. However, for regional networks, the effects of satellite geometry relative to each receiver will cancel out, leaving the instantaneous difference in OTL 


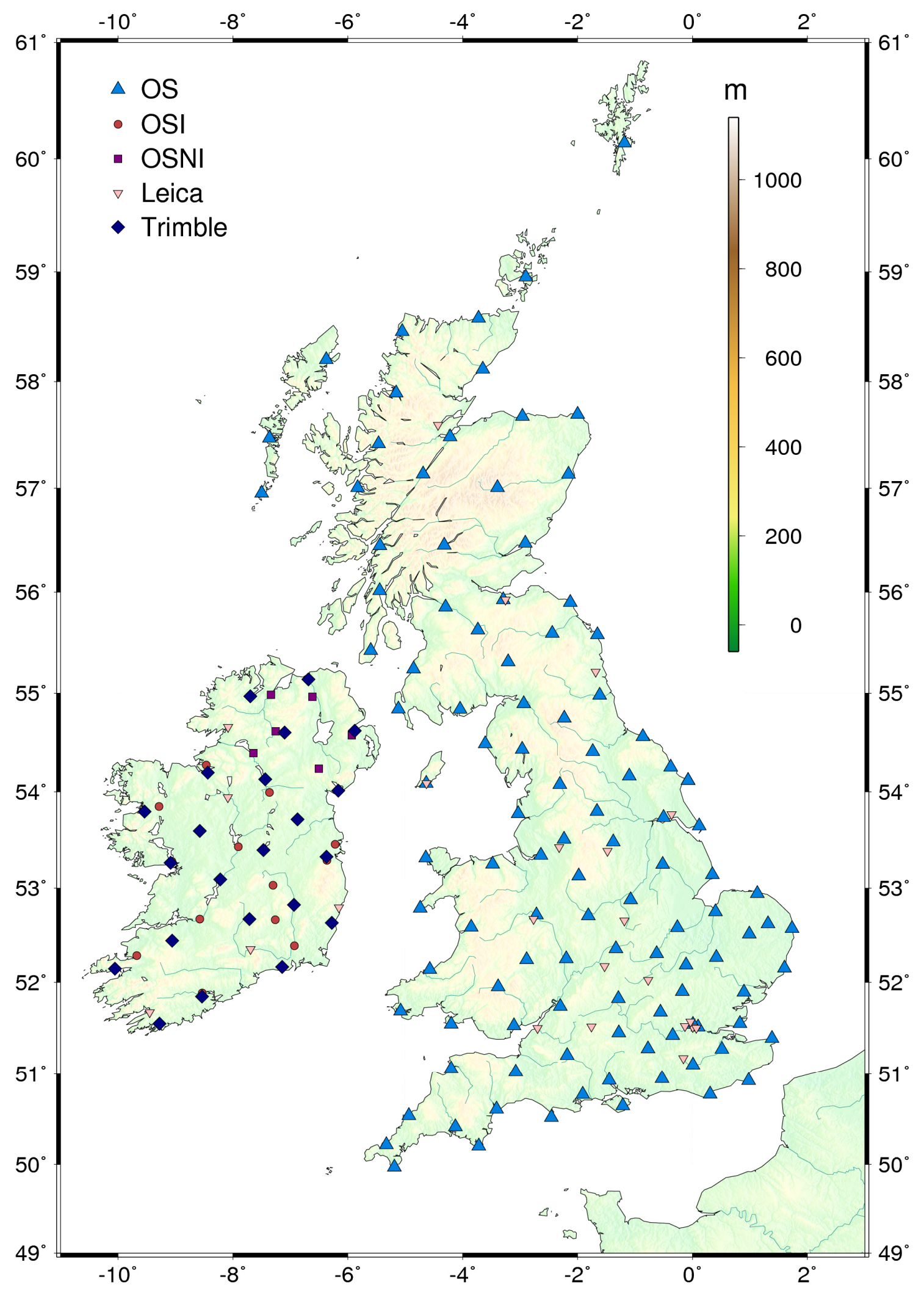

Fig. 2. Continuously operating reference stations operated by the national mapping agencies Ordnance Survey (triangles), Ordnance Survey Ireland (circles), and Ordnance Survey Northern Ireland (squares). Additional sites forming part of Leica SmartNet (inverted triangles) and Trimble VRS Now (diamonds) are also shown. 
displacement as the only significant factor (misalignment of the local topocentric coordinate systems at each site will also be negligible). Here, we investigate this residual OTL-related error as a function of position within the British Isles, for singlebaseline and network GNSS solutions. We use a range of interpolation schemes to simulate the network adjustment and error modelling processes that could occur in a network or multiple-baseline solution that does not explicitly model OTL effects. These interpolation schemes will not exactly predict the residual OTL displacement error arising from a particular (usually proprietary) algorithm, but the range of predicted errors will give a reasonable estimate of the likely magnitude of these errors and their spatial variability. Throughout, we use the FES2004 numerical ocean tide model [12], as this is representative of the latest generation of tidal models which show reasonably close agreement with each other, in most areas [e.g. 2, 11]. Site displacements are predicted using the SPOTL software [7] with Green's functions based on the accepted Gutenberg-Bullen A solid Earth average rheology [6]. For conciseness we consider only the geometry of the Trimble VRS network which provides even reference station coverage throughout the British Isles; the Leica SmartNet network gives broadly similar results.

In general, we can write the "true" OTL displacement $w$ in some direction at a point $\mathbf{x}$ (projection coordinates $x, y$, or latitude $\Phi$, longitude $\lambda$ ), caused by tidal constituent $k$ with amplitude $A_{k}$ and phase lag $\phi_{k}$, as

$$
\begin{aligned}
w_{k}(\mathbf{x}) & =A_{k} e^{i \phi_{k}}=A_{k} \cos \phi_{k}+i A_{k} \sin \phi_{k} \\
& =u_{k}+i v_{k}
\end{aligned}
$$

For simplicity, we omit the time dependence as we are concerned only with the correct amplitude and Greenwich phase lag of the signal. This "true" displacement could in principle be computed using a sufficiently accurate numerical tide model. Similarly, the OTL displacement at that point estimated from the "true" displacements at nearby reference points, can be expressed as

$$
\begin{aligned}
w_{k}^{\prime}(\mathbf{x}) & =A_{k}^{\prime} \cos \phi_{k}^{\prime}+i A_{k}^{\prime} \sin \phi_{k}^{\prime} \\
& =u_{k}^{\prime}+i v_{k}^{\prime}
\end{aligned}
$$

where the estimation is achieved separately for the real and imaginary parts. The residual OTL displacement at that point is therefore given by the difference between the "true" and estimated OTL displacements:

$$
\begin{aligned}
\widetilde{w}_{k}(\mathbf{x}) & =\left(u_{k}-u_{k}^{\prime}\right)+i\left(v_{k}-v_{k}^{\prime}\right) \\
& =\widetilde{A}_{k} \cos \widetilde{\phi}_{k}+i \widetilde{A}_{k} \sin \widetilde{\phi}_{k}
\end{aligned}
$$

Here, we consider the eight major tidal constituents $k \in\{\mathrm{M} 2, \mathrm{~S} 2, \mathrm{~N} 2, \mathrm{~K} 2, \mathrm{~K} 1, \mathrm{O} 1$, P1, Q1\} (Table 1), and also the summed semi-diurnal and summed diurnal residual amplitudes which represent the maximum variation from the true coordinate over these timescales respectively. It should be noted that the total maximum semi-diurnal and diurnal variation from the true coordinates is slightly less than the total of the separate semi-diurnal and diurnal residual sums, because of fixed phase relationships between some of the semi-diurnal and diurnal tidal constituents. 

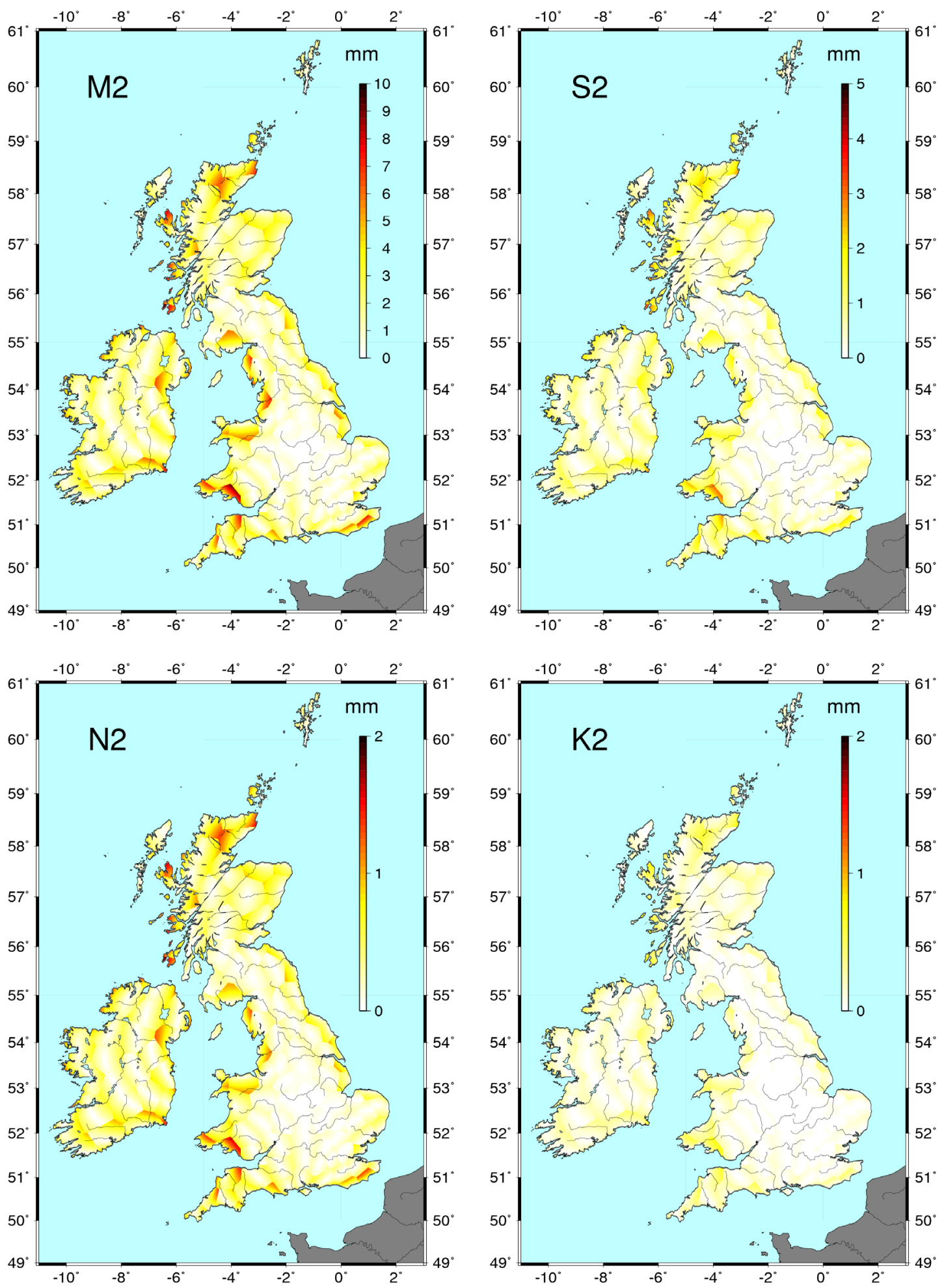

Fig. 3. Residual vertical OTL displacement complex amplitude with respect to the nearest CORS (semi-diurnal constituents): M2 top left, S2 top right, N2 bottom left, K2 bottom right. Note that the amplitude scales for M2 and S2 differ from each other and are larger than those for all other constituents. 

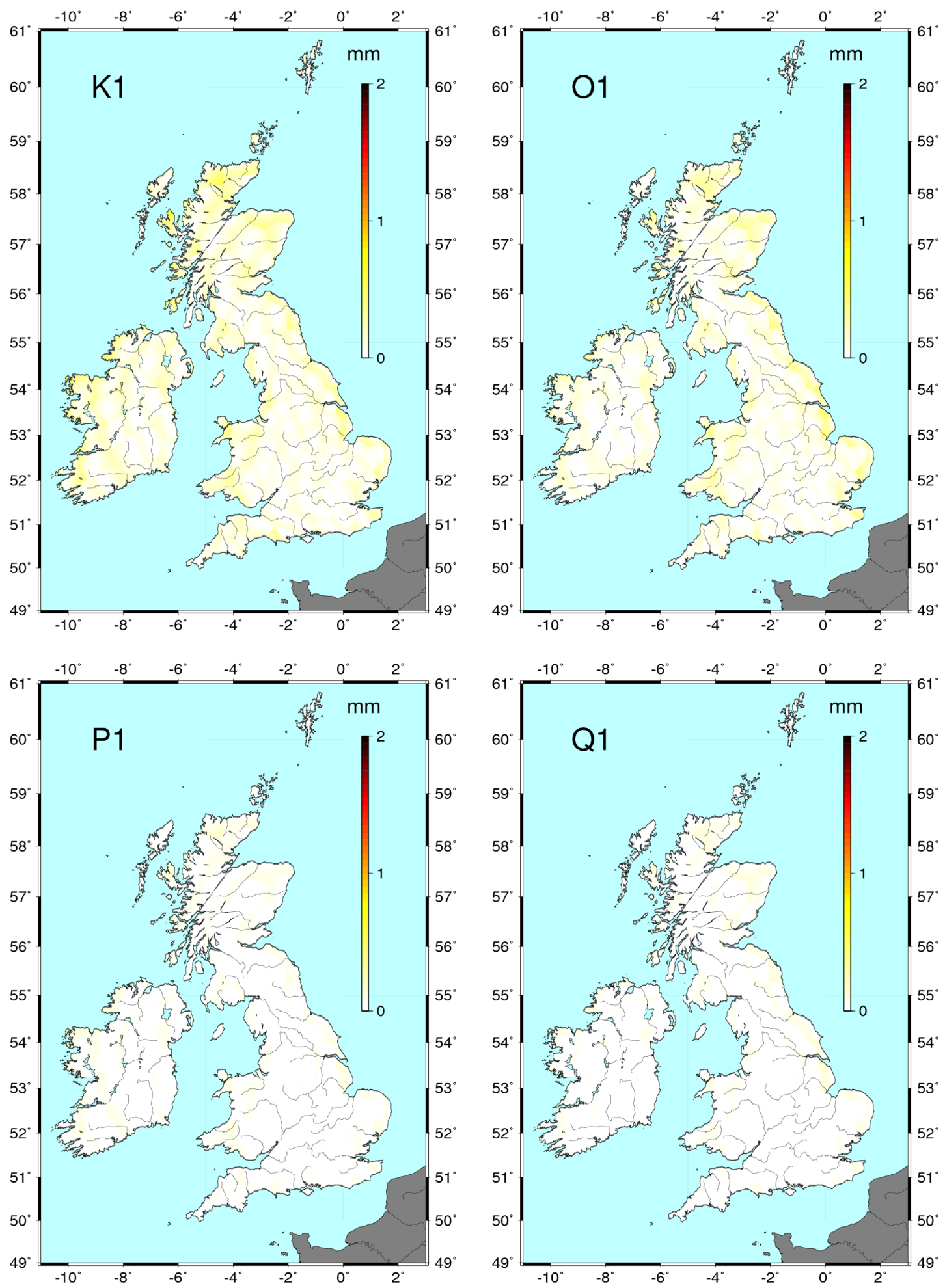

Fig. 4. Residual vertical OTL displacement complex amplitude with respect to the nearest CORS (diurnal constituents): $\mathrm{K} 1$ top left, $\mathrm{O} 1$ top right, $\mathrm{P} 1$ bottom left, Q1 bottom right. 


\section{Single-baseline GNSS}

For single-baseline solutions, the estimated OTL displacement at the rover is simply that at the reference station, taken to be the nearest CORS in the network (location $\mathbf{x}_{0}$ ),

$$
w_{k}^{\prime}(\mathbf{x})=w_{k}\left(\mathbf{x}_{0}\right)
$$

and so the residual OTL displacement is the difference between the "true" (computed) OTL displacement at the rover location and that at the nearest CORS. Residual complex amplitudes of vertical OTL displacement for each tidal constituent are shown in Figures 3 and 4 . In general, the effects of the diurnal OTL constituents are mitigated more successfully than the semi-diurnal. This is not merely because the absolute diurnal OTL displacements are smaller; it is also because their spatial variations in both amplitude and phase are smoother and so the relative OTL displacement is correspondingly reduced. By far the majority of the residual OTL effects are due to the M2 constituent, although S2 and N2 are also considerable in places. For all constituents, residual OTL is greatest towards the margins of the cells centred on each CORS, and discontinuities in residual OTL exist at these margins. Summed residual vertical complex amplitudes for the semi-diurnal and diurnal constituents are shown in Figure 5. The former are greater than $5 \mathrm{~mm}$ throughout much of the British Isles, except central England. The summed horizontal component amplitudes of residual OTL displacement (Figure 6), which are dependent on the lateral gradients of the load potential, are generally smaller. However, the summed complex amplitudes of the residual semi-diurnal OTL displacements are greater than $1 \mathrm{~mm}$ in the horizontal in most of these same areas. These biases, particularly in the vertical, are of only slightly smaller magnitude than the typical errors in baseline and Network RTK GNSS positioning [21, 22], and are even more significant in proportion to the positional uncertainty of short-occupation or rapid static relative GNSS.

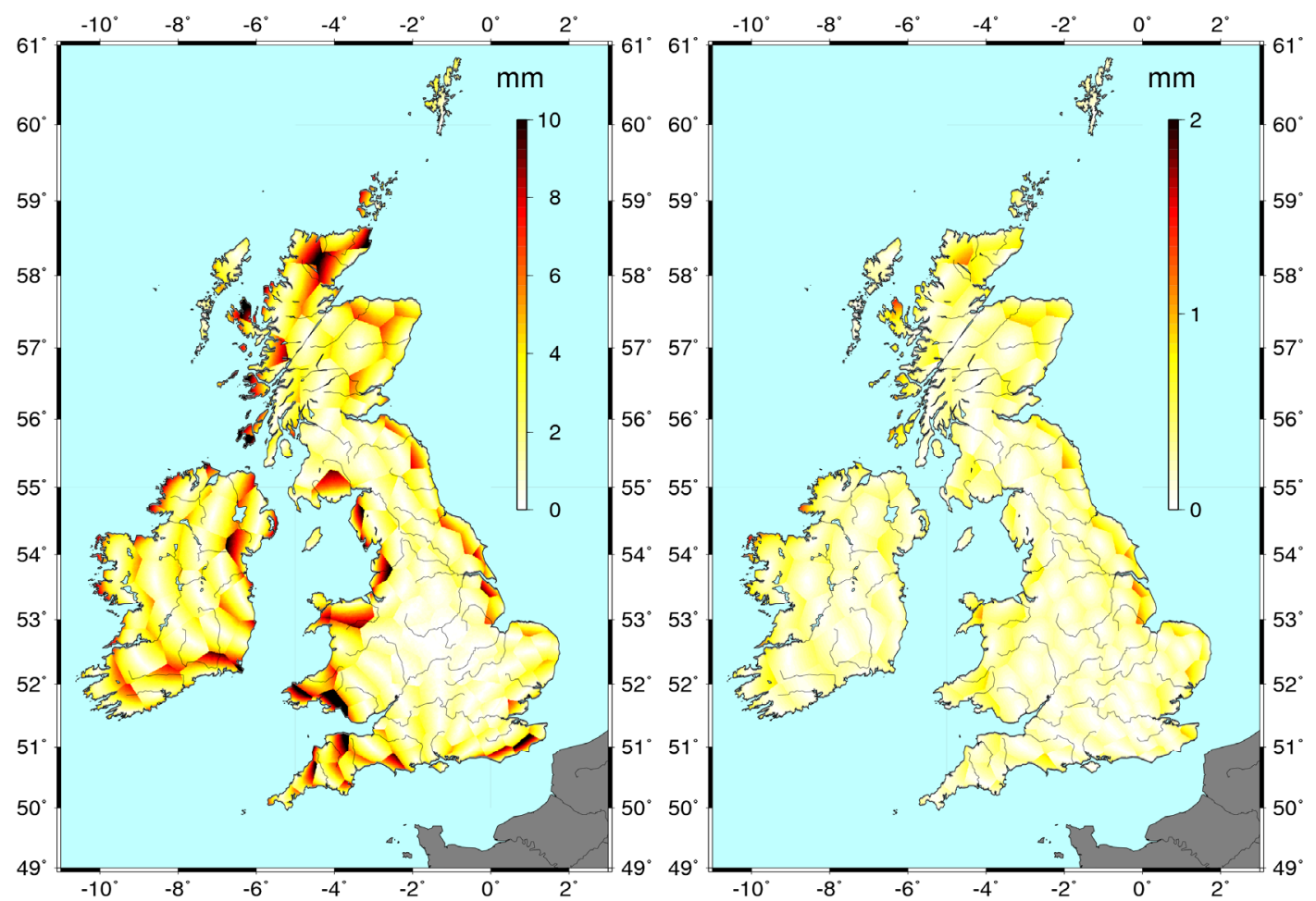

Fig. 5. Summed complex amplitudes of semi-diurnal (left) and diurnal (right) residual vertical OTL displacement with respect to the nearest CORS. 


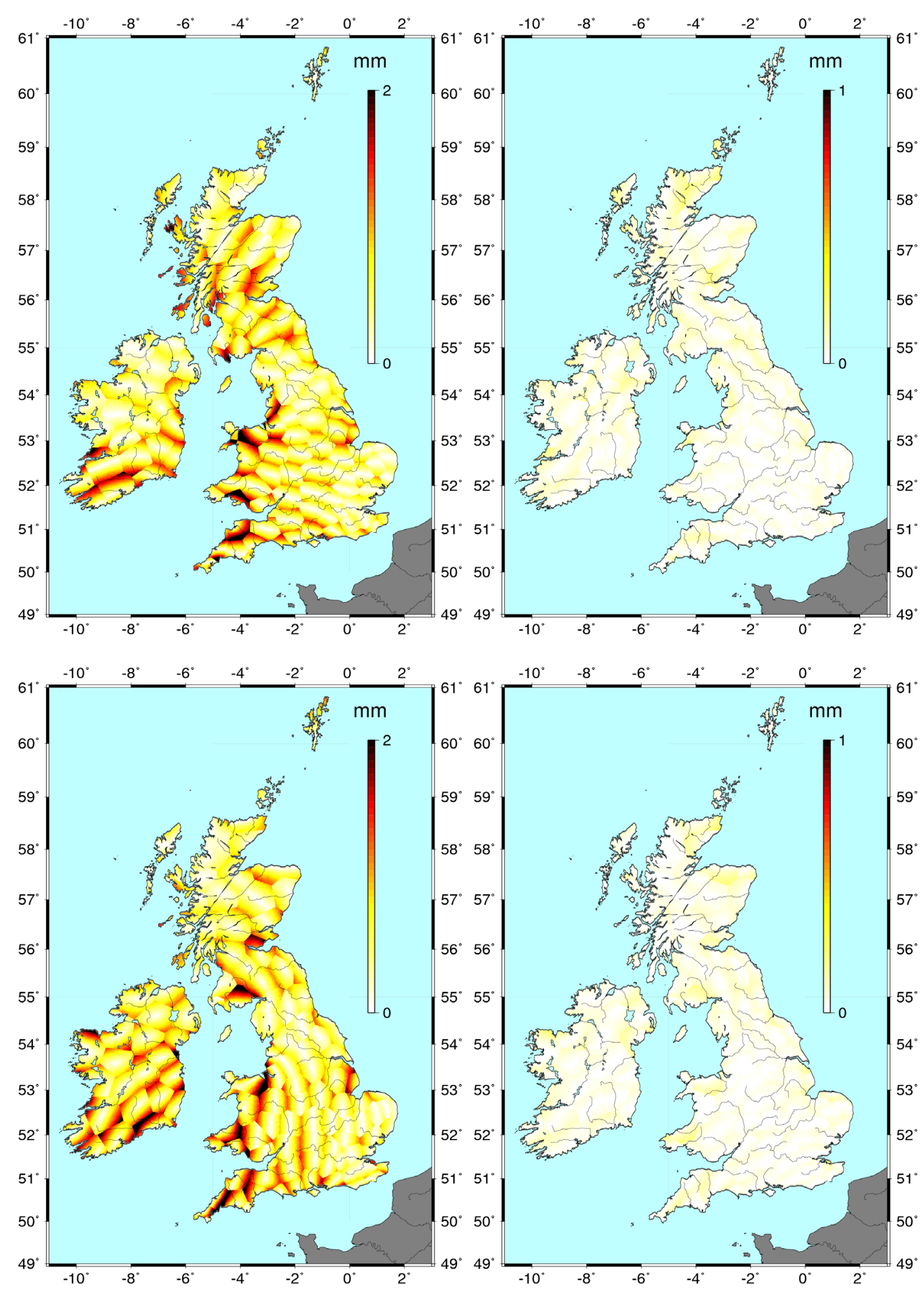

Fig. 6. Summed complex amplitudes of semi-diurnal (left) and diurnal (right) residual lateral OTL displacement with respect to the nearest CORS: (top) east component, and (bottom) north component. 


\section{Network GNSS}

In a generic network GNSS solution, error models can be interpolated to the rover location in several ways, depending on the number and arrangement of reference stations used. Firstly, an exact planar fit could be obtained to the residuals at the three nearest sites, which could be interpolated (if these sites surround the rover) or extrapolated (if they do not) to the rover location. Secondly, an exact planar fit could be obtained using the three sites bounding the rover; this will always result in an interpolation, but no values will be available outside of the Delaunay triangulation of the reference station network. Thirdly, an exact bilinear fit could be obtained to the residuals at the nearest four sites and interpolated or extrapolated from this quadrilateral to calculate the error at the rover. Of course, more complicated higherorder fitting schemes are also possible, as are over-determined planar or bilinear fits (using more than three or four reference stations respectively), but for simplicity we do not consider these here.

For each of the real and imaginary components $z=\left\{u_{\mathrm{M} 2}, v_{\mathrm{M} 2}, u_{\mathrm{S} 2}, v_{\mathrm{S} 2}, \ldots u_{k}, v_{k}\right\}$ of OTL displacement in a coordinate direction, due to some tidal constituent $k$, the planar fit at point $\mathbf{x}$ to the displacements at three nearby reference stations with coordinates $\mathbf{x}_{0}, \mathbf{x}_{1}$ and $\mathbf{x}_{2}$ (and component values $z_{0}, z_{1}, z_{2}$ ) is given exactly by

$$
z^{\prime}=\frac{1}{x_{1} y_{2}-x_{2} y_{1}}\left(\begin{array}{c}
z_{1}-z_{0} \\
z_{2}-z_{0}
\end{array}\right)^{T}\left(\begin{array}{cc}
y_{2} & -x_{2} \\
-y_{1} & x_{1}
\end{array}\right)\left(\begin{array}{l}
x \\
y
\end{array}\right)+z_{0}
$$

with all coordinates expressed relative to a local origin at vertex $\mathbf{x}_{0}$. The denominator represents a degeneracy condition on the triangle of reference sites. This result follows from the fact that the component values at each vertex obey the coplanarity condition

$$
z_{i}=a+b x_{i}+c y_{i}
$$

which leads to

$$
\left(\begin{array}{l}
z_{1} \\
z_{2}
\end{array}\right)=\left(\begin{array}{l}
z_{0} \\
z_{0}
\end{array}\right)+\left(\begin{array}{ll}
x_{1} & y_{1} \\
x_{2} & y_{2}
\end{array}\right)\left(\begin{array}{l}
b \\
c
\end{array}\right)
$$

from which the result follows by matrix inversion. Equation 5 can be evaluated using either the three nearest sites to the point, or the three sites forming the vertices of the Delaunay triangle bounding the point. For the first of these cases, maximum residual vertical OTL displacements are shown in Figure 7. In many areas, these are smaller than $2 \mathrm{~mm}$, but there are a number of regions where they reach this level and even some localised pockets where the residual signal can exceed $10 \mathrm{~mm}$. For the most part, these larger excursions occur where the base station data are being extrapolated, as can be seen from the lack of such features in the interpolated OTL displacements using the Delaunay triangulation (Figure 8). However, this interpolation is neither able to prevent errors of order $5 \mathrm{~mm}$ in south-west Wales, south-west England, Kent, and Dumfries and Galloway; nor able to provide any OTL corrections in the Shetland Islands, Western Isles, or parts of western Ireland. 


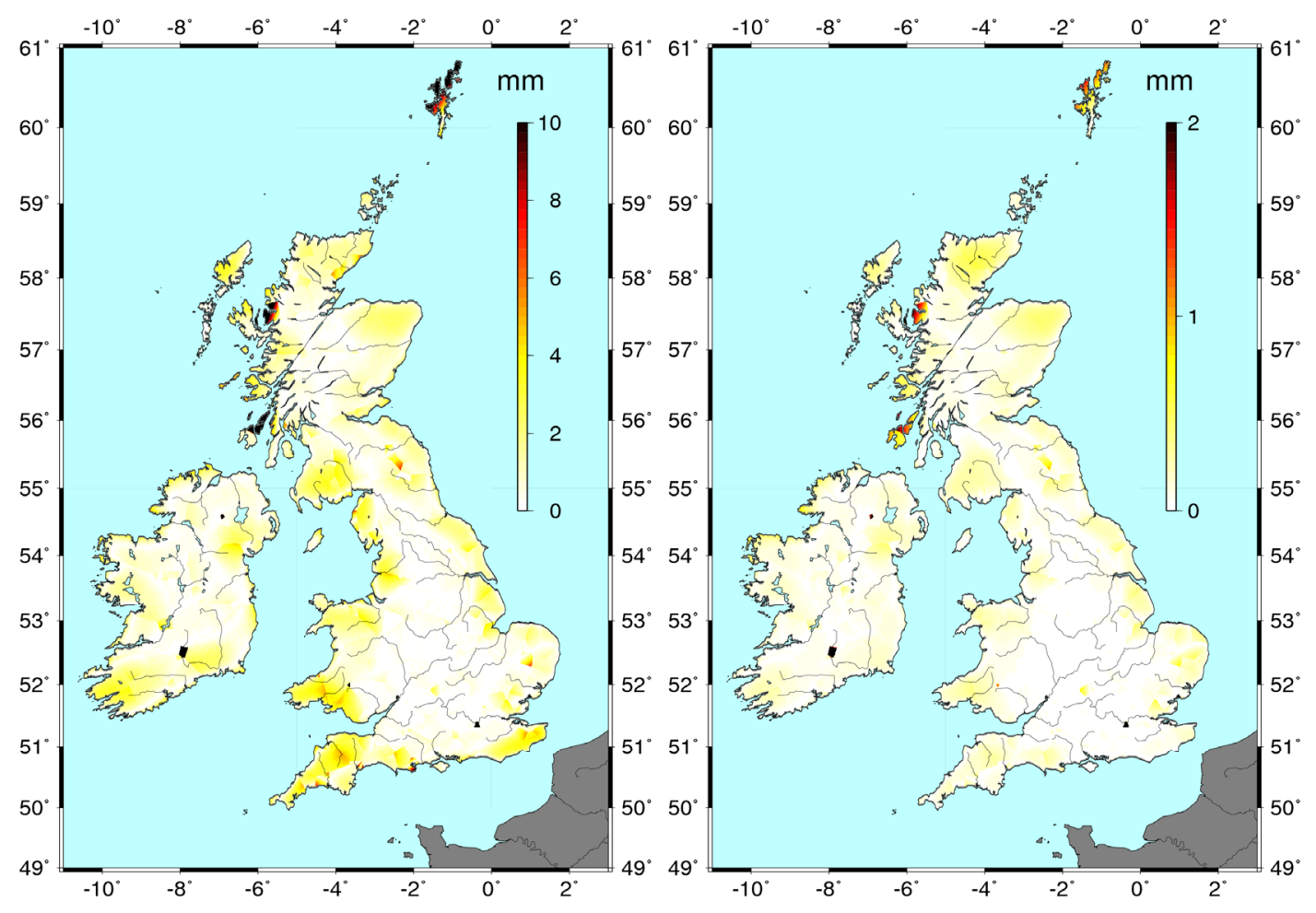

Fig. 7. Summed complex amplitudes of semi-diurnal (left) and diurnal (right) residual vertical OTL displacement with respect to a planar estimate using the nearest three CORS locations.

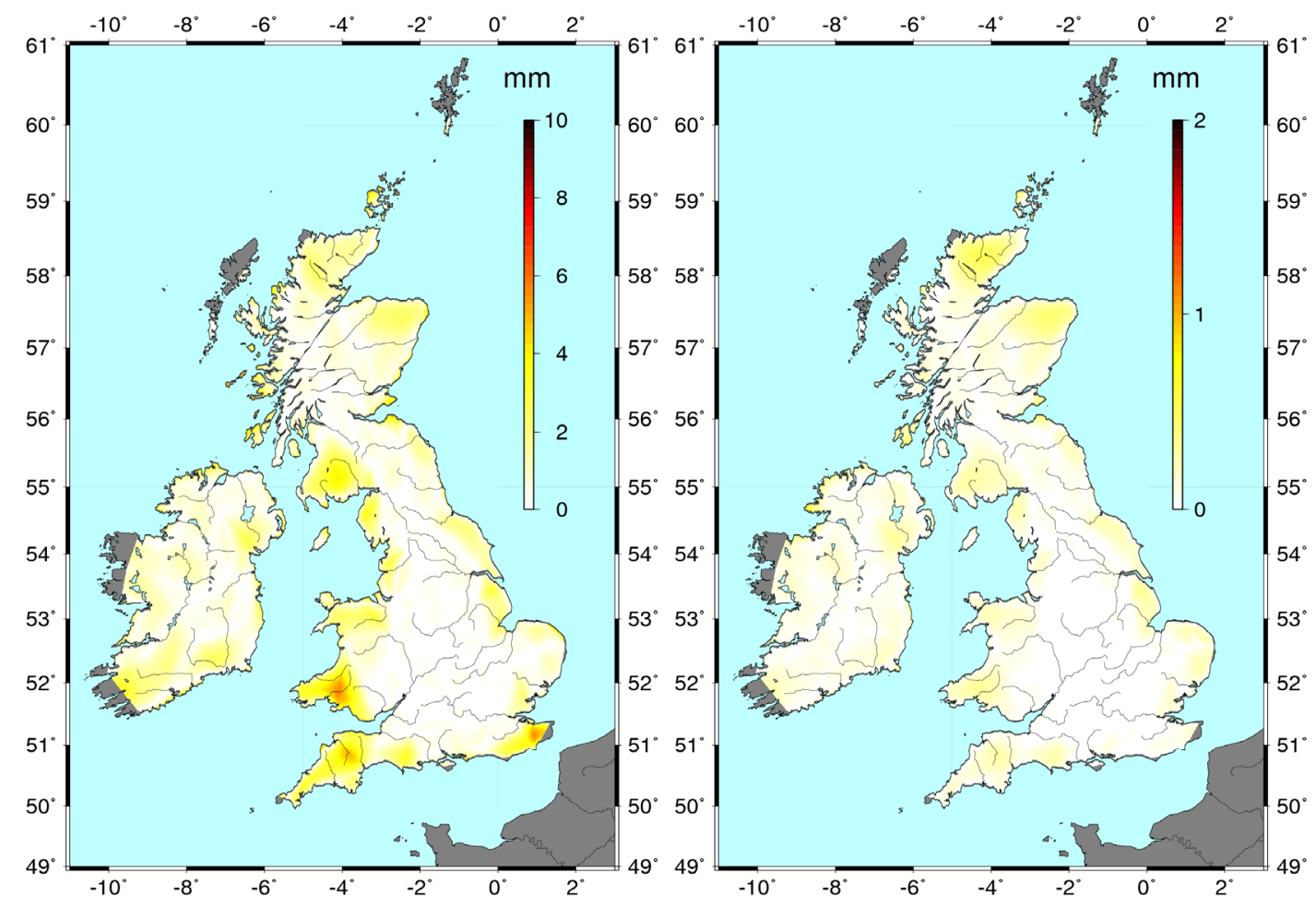

Fig. 8. Summed complex amplitudes of semi-diurnal (left) and diurnal (right) residual vertical OTL displacement with respect to a planar estimate using the three CORS locations forming the Delaunay triangle bounding the point. 
Bilinear interpolation should in principle provide better corrections in regions that are well served with reference stations. In general, bilinear interpolation is carried out on a regular, i.e. square or rectangular, grid [e.g. 23]. We have derived a closed form for the bilinear interpolation of the real or imaginary part of an OTL displacement in an irregular quadrilateral $\left(\mathbf{x}_{0}, \mathbf{x}_{1}, \mathbf{x}_{2}, \mathbf{x}_{3}\right)$, given by

$$
z^{\prime}=\frac{1}{M}\left(\begin{array}{c}
z_{1}-z_{0} \\
z_{2}-z_{0} \\
z_{3}-z_{0}
\end{array}\right)^{T}\left(\begin{array}{ccc}
\left(x_{3}-x_{2}\right) y_{2} y_{3} & x_{2} x_{3}\left(y_{2}-y_{3}\right) & x_{2} y_{3}-x_{3} y_{2} \\
\left(x_{1}-x_{3}\right) y_{1} y_{3} & x_{1} x_{3}\left(y_{3}-y_{1}\right) & x_{3} y_{1}-x_{1} y_{3} \\
\left(x_{2}-x_{1}\right) y_{1} y_{2} & x_{1} x_{2}\left(y_{1}-y_{2}\right) & x_{1} y_{2}-x_{2} y_{1}
\end{array}\right)\left(\begin{array}{c}
x \\
y \\
x y
\end{array}\right)+z_{0}
$$

where, again, all coordinates are expressed relative to a local origin at vertex $\mathbf{x}_{0}$. The derivation is analogous to that of equation 5. For equation 6 to be valid, the quantity

$$
M=x_{1} x_{3} y_{2}\left(y_{3}-y_{1}\right)+x_{2} x_{3} y_{1}\left(y_{2}-y_{3}\right)+x_{1} x_{2} y_{3}\left(y_{1}-y_{2}\right)
$$

must be non-zero, which represents a non-degeneracy condition for the quadrilateral. Here, we interpolate (or extrapolate) OTL displacements from the quadrilateral formed by the nearest four reference stations. The maximum complex amplitudes of semidiurnal and diurnal residual vertical OTL displacements are shown in Figure 9. In most locations, the performance of this bilinear interpolation scheme is slightly worse than that of the planar interpolation schemes, although better than that of nearestneighbour estimation. The largest, semi-diurnal, residuals are generally of order 0.5-2 mm, but there are numerous "dead zones" where these exceed $10 \mathrm{~mm}$. These features are not present in the planar estimation schemes, and result from the increased extrapolation error of the bilinear form.

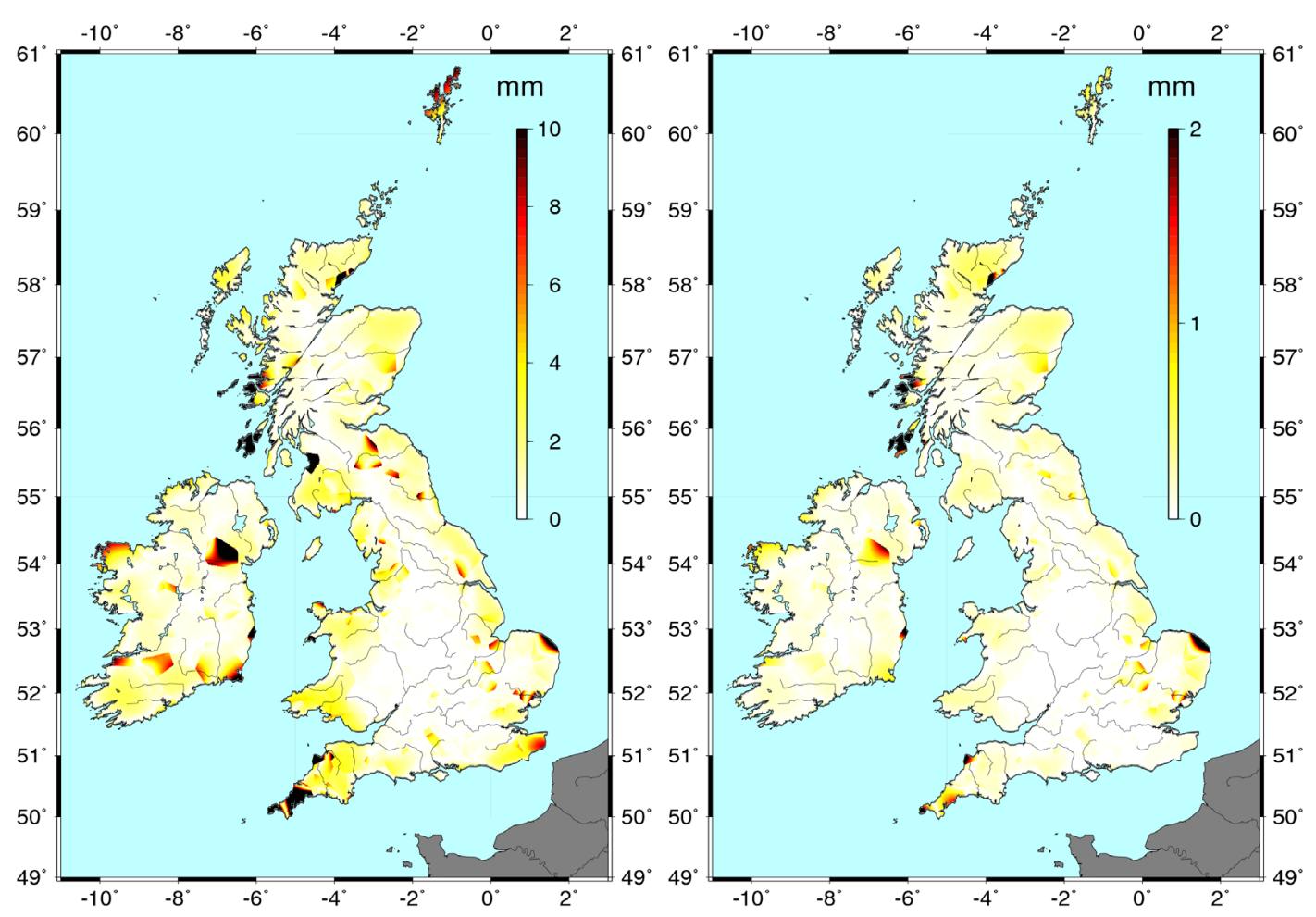

Fig. 9. Summed complex amplitudes of semi-diurnal (left) and diurnal (right) residual vertical OTL displacement with respect to a bilinear estimate using the nearest four CORSs. 


\section{ESTIMATION AND REMOVAL OF RESIDUAL OCEAN TIDE LOADING ERROR}

Depending on the error interpolation scheme used by the Network RTK solution, residual OTL displacement errors typically up to $5 \mathrm{~mm}$ but in some cases exceeding $10 \mathrm{~mm}$ will exist at points in the British Isles. The magnitude of these errors will depend not only on the constructive or destructive interference of different tidal constituents at a point, which is relatively easy to predict, but also on the geometry of the reference station network used to generate the RTK solution. Although some generalisations can be made as to the most problematic areas, the latter effect is not readily predictable; moreover, it will vary as sites are added to or removed from the network, or suffer temporary outages. The practical surveyor therefore needs some means of assessing the approximate level of residual OTL displacement error in a locality, and of mitigating it should this be necessary to achieve positioning accuracy that is fit for purpose.

To a reasonable first-order approximation, the residual OTL displacement error at a point, within a given day, can be regarded as the sum of an aggregated semi-diurnal signal (in which the M2 constituent will dominate) and a similar diurnal signal:

$$
h_{1}=h_{0}+A_{S} \cos \phi_{S}+A_{D} \cos \phi_{D}+e_{1}
$$

where $\phi_{S}$ and $\phi_{D}$ are lumped terms representing the phases of the semi-diurnal and diurnal terms at the epoch, and $A_{S}$ and $A_{D}$ are their amplitudes at the location in question. We consider here the epoch vertical coordinate $h_{1}$ and its long-term mean $h_{0}$, although the analysis could also be applied similarly in the case of the east and north coordinates which suffer from generally smaller OTL displacements. $e_{1}$ represents the unknown GNSS measurement error at this epoch, which is assumed to be drawn from a population with zero mean and standard deviation $\sigma$.

At a time just over three hours later, the semi-diurnal signal will have advanced by a quarter cycle and the diurnal signal by an eighth of a cycle, so the epoch coordinate will be:

$$
\begin{aligned}
h_{2} & =h_{0}+A_{S} \cos \left(\phi_{S}+\frac{\pi}{2}\right)+A_{D} \cos \left(\phi_{D}+\frac{\pi}{4}\right)+e_{2} \\
& =h_{0}-A_{S} \sin \phi_{S}+\frac{1}{\sqrt{2}} A_{D}\left(\cos \phi_{D}-\sin \phi_{D}\right)+e_{2}
\end{aligned}
$$

followed after another three hours (i.e. a total of just over six hours) by:

$$
\begin{aligned}
h_{3} & =h_{0}+A_{S} \cos \left(\phi_{S}+\pi\right)+A_{D} \cos \left(\phi_{D}+\frac{\pi}{2}\right)+e_{3} \\
& =h_{0}-A_{S} \cos \phi_{S}-A_{D} \sin \phi_{D}+e_{3}
\end{aligned}
$$

Taking the mean of equations 8 and 10, we obtain the quantity

$$
\begin{aligned}
\bar{h} & =\frac{1}{2}\left(h_{1}+h_{3}\right)=h_{0}+\frac{1}{2} A_{D}\left(\cos \phi_{D}-\sin \phi_{D}\right)+\frac{1}{2}\left(e_{1}+e_{3}\right) \\
& =h_{0}+\frac{1}{\sqrt{2}} A_{D} \cos \left(\phi_{D}+\frac{\pi}{4}\right)+\frac{1}{2}\left(e_{1}+e_{3}\right)
\end{aligned}
$$


Averaged over many instances and assuming that the GNSS measurement errors are uncorrelated at separations of roughly six hours, $\bar{h}$ will be an unbiased estimate of the long-term mean vertical coordinate $h_{0}$, but individual estimates of $\bar{h}$ will suffer from a small bias of up to $A_{D} / \sqrt{2}$ depending on the (for statistical analysis purposes, effectively random) initial phase of the diurnal term $\phi_{D}$. If the residual diurnal OTL displacement error is small compared with the other errors in an instantaneous or shortterm averaged coordinate measurement, this bias can be neglected. Figures 7, 8 and 9 show that this will indeed be the case for Network RTK GNSS measurements throughout the British Isles, which have typical root mean square errors of 15-30 mm in the vertical and $10-15 \mathrm{~mm}$ in plan $[\mathbf{2 1}, \mathbf{2 2}]$. Equation 11 can therefore be used to obtain site coordinates unaffected by OTL, using two sets of coordinates obtained instantaneously or from short-term averages at each survey point, separated by half the dominant semi-diurnal OTL period (i.e. roughly 6 $1 / 4$ hours). Note that this is not equivalent to a continuous "quasi-kinematic" occupation of the site over the same time-span, which suffers from operator inefficiency without improving robustness to OTL errors, nor is it equivalent single batch coordinate solution which will also suffer from propagated OTL error effects $[\mathbf{1}, \mathbf{2}, \mathbf{3}]$.

As a means of determining whether the semi-diurnal signal is large enough that this averaging is necessary, we can compute the quantity

$$
\Sigma^{2}=\left(h_{1}-\bar{h}\right)^{2}+\left(h_{3}-\bar{h}\right)^{2}=\frac{1}{2} h_{1}^{2}+h_{2}^{2}+\frac{1}{2} h_{3}^{2}-h_{1} h_{2}-h_{2} h_{3}
$$

The meaning of $\Sigma$ is evident if we consider the trivial case in which the diurnal OTL displacement error and the measurement noise are both zero:

$$
\Sigma^{2}=\left(h_{1}-\bar{h}\right)^{2}+\left(h_{3}-\bar{h}\right)^{2}=\left(A_{S} \cos \phi_{S}\right)^{2}+\left(A_{S} \sin \phi_{S}\right)^{2}=A_{S}^{2}
$$

In practice, as shown in Figures 7, 8 and 9, the diurnal OTL displacement error can indeed be neglected, but the random measurement noise cannot, so

$$
\begin{aligned}
\Sigma^{2}= & A_{S}^{2}+A_{S} \cos \phi_{S}\left(e_{1}-e_{3}\right)+A_{S} \sin \phi_{S}\left(e_{1}-2 e_{2}+e_{3}\right) \\
& +\frac{1}{2} e_{1}^{2}+e_{2}^{2}+\frac{1}{2} e_{3}^{2}-e_{1} e_{2}-e_{2} e_{3}
\end{aligned}
$$

and has an expected (mean) value, and variance about its mean, of

$$
\begin{aligned}
\mathrm{E}\left(\Sigma^{2}\right) & =A_{S}{ }^{2}+2 \sigma^{2} \\
\operatorname{Var}\left(\Sigma^{2}\right) & =4 A_{S}{ }^{2} \sigma^{2}+2 \sigma^{4}
\end{aligned}
$$

$\Sigma^{2}$ therefore provides us with a very conservative upper bound on the level of OTL displacement error at a site; if $\Sigma^{2}>>2 \sigma^{2}$ (the latter of which can be estimated from a few tens of minutes of data), then the semi-diurnal residual OTL displacement is likely to be significant compared with other errors, and averaging using equation 11 should be applied. Two comments should be noted. Firstly, because of the modulation of $A_{S}$ throughout the spring/neap and longer tidal cycles, the estimate $\Sigma^{2}$ will only be valid on a given day, or on neighbouring days but with some degradation of quality. Secondly, $\Sigma^{2}$ is a noisy estimate, so it is best to exercise caution when dispensing with coordinate averaging. 


\section{DISCUSSION}

Ocean tide loading displacement error affects GNSS coordinate estimates in all parts of the British Isles, even when mitigated by relative positioning. For single-baseline relative positioning, this will reach several millimetres in plan and exceed $10 \mathrm{~mm}$ in the vertical in many locations; only central England is as a whole immune to these error levels. Outside of central England, OTL-related error on a baseline is therefore likely to be appreciable with respect to RTK GNSS coordinate accuracy, and significant with respect to static GNSS accuracy over short sessions up to around one hour in length.

Network relative positioning will tend to mitigate OTL-related error further. We have simulated this reduction using a realistic range of estimation schemes. Vertical OTL displacement errors can be reduced to below the $5 \mathrm{~mm}$ level throughout the vast majority of the British Isles; however, pockets of higher potential error remain when the planar and bilinear estimation schemes are used to extrapolate from their defining polygons, and planar interpolation based on a Delaunay triangulation of the CORS network provides incomplete coverage especially near coasts. It cannot therefore be assumed that network positioning will always provide better results than baseline positioning, even though this is often true.

Because the diurnal constituents of OTL displacements vary more smoothly, these errors are more effectively mitigated by all relative positioning algorithms, whether using baselines or networks. The level of the dominant residual semi-diurnal error in a locality on a given day can be conservatively estimated using three short-term measurements at a test point spread evenly over a $6-6 \frac{1}{2}$ hour period, and if necessary eliminated using two such measurements separated by this interval at each survey point (other points can be observed in the interim). This is an efficient strategy which allows large numbers of points to be observed with reasonable accuracy.

Despite all of these mitigation and adaptation strategies, the only way to guarantee that OTL-related error is efficiently reduced below the few millimetre level throughout the British Isles is to incorporate models of OTL displacement into GNSS processing at the observation level, as is done in scientific GNSS software for precise point positioning and relative processing. As shown in Figure 1, the level of agreement between OTL displacements predicted from modern numerical tide models is sufficient to allow such corrections to be made at a level suitable for routine GNSS processing in engineering survey applications.

\section{ACKNOWLEDGEMENTS}

This work originated from discussions held during the development of the Best Practice Guidance Notes for Network RTK Surveying in Great Britain by Newcastle University, under the auspices of The Survey Association. We would like to thank all stakeholders in that project (The Survey Association, Ordnance Survey, Leica Geosystems, Trimble, and the Royal Institution of Chartered Surveyors) for their initial input, and in particular Stuart Edwards (Newcastle University) for his encouragement and comments during the later work. Matt King provided helpful comments on a draft of this manuscript. Figures were produced using the publicdomain Generic Mapping Tools software [24]. 


\section{References}

1. Stewart, M. P., Penna, N. T. and Lichti, D. D., 2005. Investigating the propagation mechanism of unmodelled systematic errors on coordinate time series estimated using least squares. J. Geodesy, 79: 479-489.

2. Penna, N. T., King, M. A. and Stewart, M. P., 2007. GPS height time series: Short period origins of spurious long-period signals. J. Geophys. Res., 112: B02402.

3. King, M. A., Watson, C. S., Penna, N. T. and Clarke, P. J., 2008. Subdaily signals in GPS observations and their effect at semiannual and annual periods. Geophys. Res. Lett., 35: L03302.

4. Zumberge, J. F., Heflin, M. B., Jefferson, D. C., Watkins, M. M. and Webb, F. H., 1997. Precise point positioning for the efficient and robust analysis of GPS data from large networks. J. Geophys. Res., 102(B3): 5005-5017.

5. Agnew, D. C., Earth tides. In Treatise on Geophysics, ed. G. Schubert, 3, 163195, Elsevier, Amsterdam.

6. Farrell, W. E., 1972. Deformation of Earth by surface loads. Rev. Geophys. Space Phys., 10(3): 761-797.

7. Agnew, D. C., 1997. NLOADF: A program for computing ocean-tide loading. J. Geophys. Res., 102(B3): 5109-5110.

8. Matsumoto, K., Sato, T., Takanezawa, T. and Ooe, M., 2001. GOTIC2: a program for computation of oceanic tidal loading effect. J. Geod. Soc. Japan, 47: 243-248.

9. Bos, M. S. and Baker, T. F., 2005. An estimate of the errors in gravity ocean tide loading computations. J. Geodesy, 79(1-3): 50-63.

10. Scherneck, H.-G., 1991. A parameterized solid earth tide model and ocean tide loading effects for global geodetic base-line measurements. Geophys. J. Int., 106(3): 677-694.

11. Penna, N. T., Bos, M. S., Baker, T. F. and Scherneck, H.-G., 2008. Assessing the accuracy of predicted ocean tide loading displacement values. J. Geodesy, 82(12): 893-907.

12. Lyard, F., Lefévre, F., Letellier, T. and Francis, O., 2006. Modelling the global ocean tides: Modern insights from FES2004. Ocean Dynam., 56(5-6): 394-415.

13. Eanes, R. J. and Bettadpur, S., 1996. The CSR3.0 global ocean tide model: diurnal and semi-diurnal ocean tides from TOPEX/POSEIDON altimetry. Report CSR-TM-96-05, The University of Texas, Center for Space Research.

14. Ray, R. D., 1999. A global ocean tide model from TOPEX/POSEIDON altimetry: GOT99.2. NASA Tech. Memo TM-209478, 58 pp.

15. Matsumoto, K., Takanezawa, T. and Ooe, M., 2000. Ocean tide models developed by assimilating TOPEX/POSEIDON altimeter data into hydrodynamical model: a global model and a regional model around Japan. J. Oceanogr., 56: 567-581.

16. Egbert, G. D. and Erofeeva, S. Y., 2002. Efficient inverse modeling of barotropic ocean tides. J. Atm. Oceano. Tech., 19(2): 183-204.

17. Khan, S. A. and Scherneck, H.-G. The M2 ocean tide loading wave in Alaska: vertical and horizontal displacements, modelled and observed. J. Geodesy, 77: $117-127$.

18. Vollath, U., Buecherl, A., Landau, H., Pagels, C. and Wagner, B., 2000. Multibase RTK positioning using virtual reference stations. Proc. ION GPS2000, 123131.

19. Fotopoulos, G. and Cannon, M. E., 2001. An overview of multi-reference station methods for cm-level positioning. GPS Solutions, 4(3): 1-10. 
20. Wei, E., Chai, H., An, Z. and Liu, J., 2006. VRS virtual observations generation algorithm. J. Global Positioning Systems, 5(1-2): 76-81.

21. Edwards, S. J., Clarke, P. J., Goebell, S. and Penna, N. T., 2008. An examination of commercial network RTK GPS services in Great Britain. Report for The Survey Association, available at http://www.tsa-uk.org.uk/.

22. Edwards, S. J., Clarke, P. J., Penna, N. T. and Goebell, S., in press. An examination of Network RTK GPS services in Great Britain. Survey Review, this issue.

23. Press, W. H., Teukolsky, S. A., Vettering, W. T. and Flannery, B. P., 1992. Numerical Recipes in $C$ ( $2^{\text {nd }}$ edition). Cambridge University Press, Cambridge.

24. Wessel, P. and Smith, W. H. F., 1991. Free software helps map and display data, EOS Trans. $A G U, 72: 441$. 